\title{
3D Composition Imaging Using a Dedicated FIB/STEM System
}

T. Yaguchi*, M.Konno*, T. Kamino*, K. Kaji**, T.Ohnishi**, M.Watanabe***,

*Hitachi Science Systems, Ltd., 11-1, Ishikawa-cho, Hitachinaka, Ibaraki, 312-0057 Japan

**Hitachi High-Technologies Corp., 882, Ichige, Hitachinaka, Ibaraki, 312-8504 Japan

*** Dept. of Materials Science \& Engineering, Lehigh University, Bethlehem, PA,18015 USA

A technique for forming a sub-micron-square pillar specimen and then observing the sample from different directions has been developed using a focused ion beam (FIB) / scanning transmission electron microscopy (STEM) system. The system employs an FIB/STEM compatible specimen holder with a specially designed rotation mechanism, which allows the specimen to be rotated 360 degrees [1]. This technique was used for the three dimensional (3D) elemental mapping of a Ni-base superalloy specimen which contains fine $\gamma$ ' precipitates. Fig.1(a) shows a bright-field (BF)-STEM image of the cone shaped Ni-base superalloy specimen. Energy dispersive X-ray spectrometry (EDX) compositional maps of Ni-K, Cr-K and Ti-K (shown in Fig.1 (b), (c) and (d), respectively) were obtained from the same field of view as Fig.1 (a). Spectrum imaging combined with multivariate statistical analysis (MSA) [2] was used to enhance the weak X-ray signals of the 10 to $20 \mathrm{~nm}$ precipitates, which contain a low concentration of Ti-K. A rendering technique was also applied to the composition maps of Ni-K, $\mathrm{Cr}-\mathrm{K}$ and Ti-K for 3D reconstruction of fine precipitates [3].

In a second study, this technique was used for the observation of a Si device. A specimen which included a contact plug of the Si device was trimmed into a micro-pillar with a $400 \mathrm{~nm}^{2}$ cross-section and a $5 \mu \mathrm{m}$ length. Analysis was performed with a $200 \mathrm{kV}$ HD-2300 STEM equipped with the EDAX genesis system. EDX spectrum images were acquired for a dwell time of $64 \mathrm{~ms}$ per pixel with the incident beam size of $1 \mathrm{~nm}$ and the current of $0.9 \mathrm{nA}$. The sizes of spectrum images are 128 x 100 pixels with 1024 channels. Fig.2 shows a BF-STEM image (a) and a high angle annular dark-field (HAADF)-STEM image (b) of the contact plug. Fig.3 shows original EDX elemental maps of Si-K (a), Ti-K(b), N-K(c) and As-K(d). The elemental maps reconstructed from the maps shown in Fig.3 by MSA are shown in Fig.4 (a), (b), (c) and (d), respectively. The distributions of elements, especially the dopant As, were successfully enhanced by MSA. In these acquisition conditions, 36 tilt series of EDX spectrum images were acquired with a 5 degree step and then the 3D distributions of those elements were observed.

\section{References}

[1] T. Yaguchi et al. Microsc. Microanal. 9 (Suppl .2) (2003) 118-119.

[2] E.R. Malinowski, Factor Analysis in Chemistry 3rd ed., Wiley (2002).

[3] T. Yaguchi et al., Microsc. Microanal. 10 (Suppl .2) (2004) 1030-1031. 

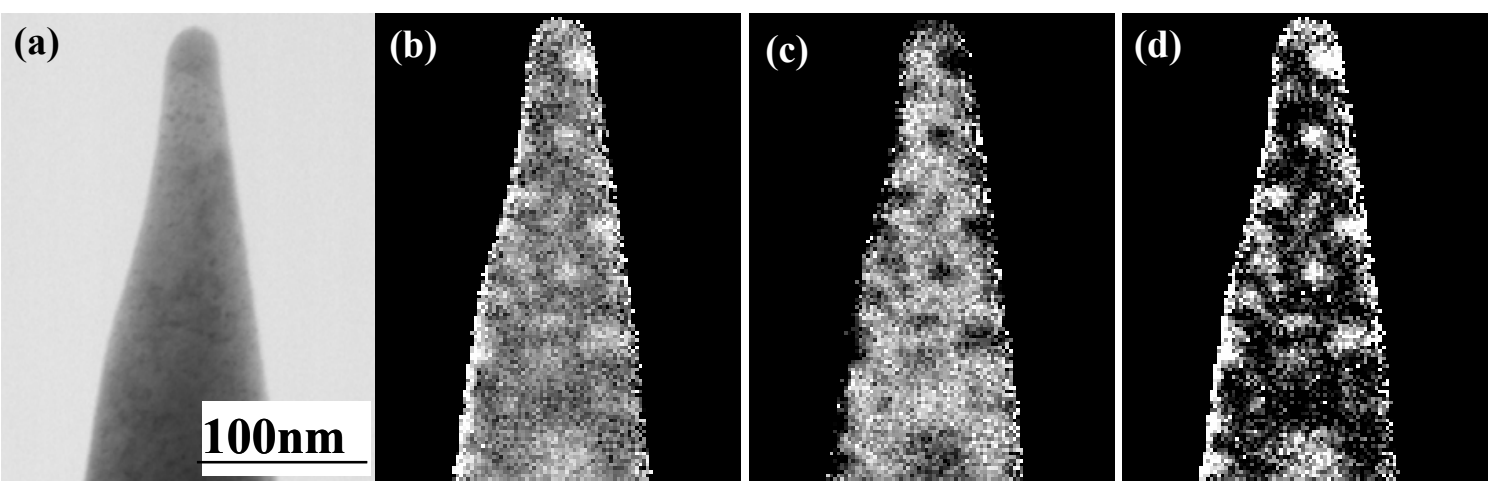

Fig.1 A BF-STEM image (a) and EDX compositional maps of Ni K (b), Cr K (c) and Ti K (c) lines from the pillar specimen in the Ni-base superalloy.
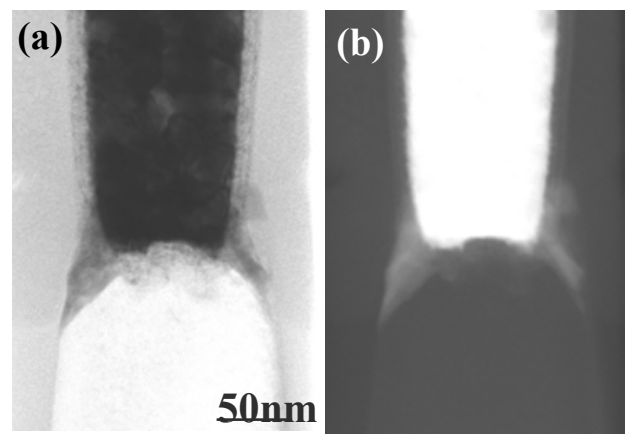

Fig.2 A BF-STEM image (a) and A HAADF-STEM image (b) of the $400 \mathrm{~nm}$ squared-pillar specimen around the contact plug in a $\mathrm{Si}$ device.
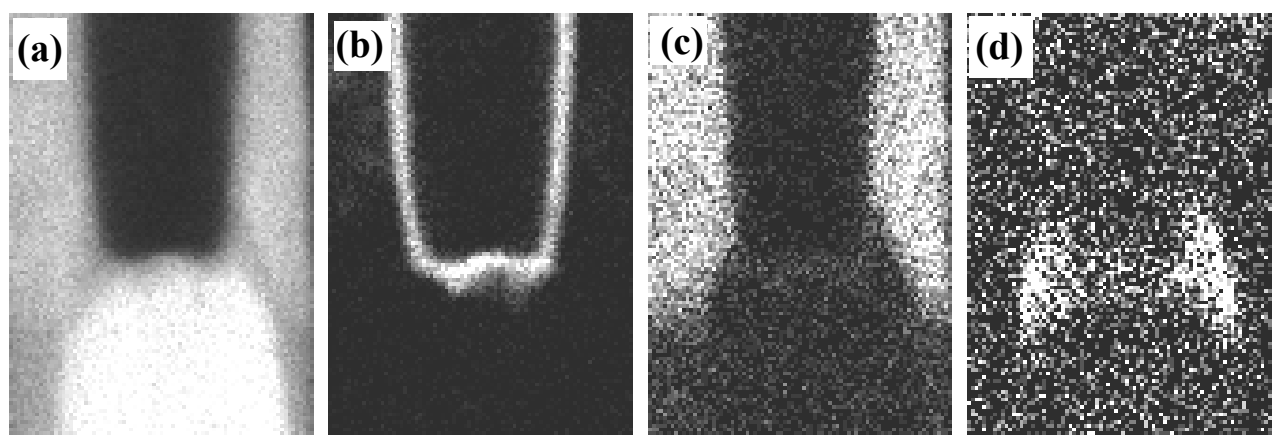

Fig.3 Original EDX elemental maps of Si K (a), Ti K (b),N K(c) and As K lines from the $400 \mathrm{~nm}$ squared-pillar specimen around the contact plug.
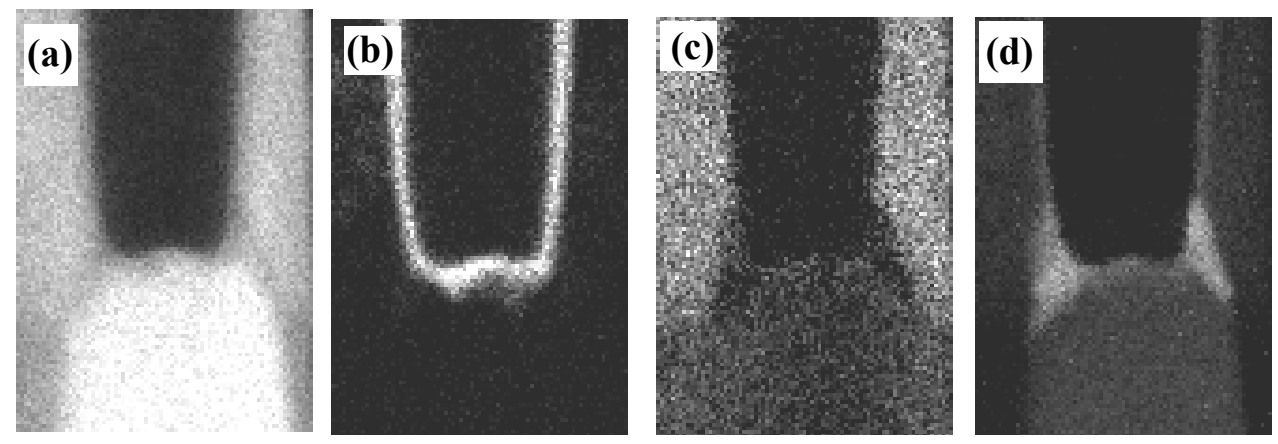

Fig.4 MSA-reconstructed EDX elemental maps of Si K (a), Ti K (b),N K(c) and As $\mathrm{K}$ lines from the $400 \mathrm{~nm}$ squared-pillar specimen around the contact plug. 